\title{
Hallux Limitus and Dynamic Splinting: A Retrospective Series
}

\author{
by Stanley R. Kalish, DPM, FACFAS ${ }^{1}$, F. Buck Willis, PhD $^{2}$ 四
}

The Foot and Ankle Online Journal 2 (4): 1

Hallux limitus $(H L)$ is commonly seen following contusions or surgical procedures at the metatarsal joint of the great toe. The purpose of this retrospective study was to examine dynamic splinting for treating HL. Sixtyone cases were examined to measure difference between HL from contusion, bunionectomy, or cheilectomy. After a mean duration of 4.2 weeks in treatment with the metatarsal dynasplint (MDS), there was a significant change for all patients $(P<0.0001)$ with a mean $73 \%$ gain in dorsiflexion at the metatarsal joint. The lack of difference between groups ( $P>0.005)$ shows the consistent benefit of MDS for HL contracture reduction.

Key words: Hallux limitus, dynamic splinting, first metatarsophalangeal joint

Accepted: March, 2009

Published: April, 2009

This is an Open Access article distributed under the terms of the Creative Commons Attribution License. It permits unrestricted use, distribution, and reproduction in any medium, provided the original work is properly cited. @The Foot and Ankle Online Journal (www.faoj.org)

$\mathrm{O}$ nset of Hallux Limitus (HL) commonly affects patients after contusion injuries and after one of the two most common surgical procedures for foot pathologies, a bunionectomy or a cheilectomy. ${ }^{1-3}$ HL is a pathology of degenerative arthritis in the first metatarsophalangeal joint (MTJ) of the first toe, or a consequence of a runner's injury this pathology affects both adolescents and adults. ${ }^{2-8}$ The literature consensus has long assumed that pain reduction is a predictable result of properly selected surgical patients, but that improvement in the arc of functional motion about the great toe should not be expected.

Address correspondence to: $\mathrm{F}$. Buck Willis, $\mathrm{PhD}$

Email: BuckPhD@yahoo.com

\footnotetext{
${ }^{1}$ Atlanta Foot and Leg Clinic, P.A.

${ }^{2}$ Texas State University (at the time of this study) and corresponding author. Dynasplint Systems Clinical Research.
}

Before HL repair with cheilectomy, radiographs typically reveal osteophyte formation in addition to dorsal exostosis, narrowing of the joint space, subchondral sclerosis, and flattening of the metatarsal head. Osteophyte formation is common on the first metatarsal head (both plantar and dorsal sides) causing inflammation, edema, pain, and reduced range of motion (ROM). Action of the hallux requires plantarflexion of the first metatarsal bone, which allows the proximal phalanx to dorsiflexion. This bidirectional hinge action must occur with each step. ${ }^{8,9}$ The normal smooth motion of the proximal phalanx over the head of the metatarsal is disrupted leading to painful compression of the phalanx against the head of the metatarsal as the joint is dorsiflexed.

Standard of care for HL includes orthoses, NSAIDs and home stretching exercises, but these protocols have had limited success in regaining range of motion because the stretching was not biomechanically correct and did not have measurable, dynamic tension. 


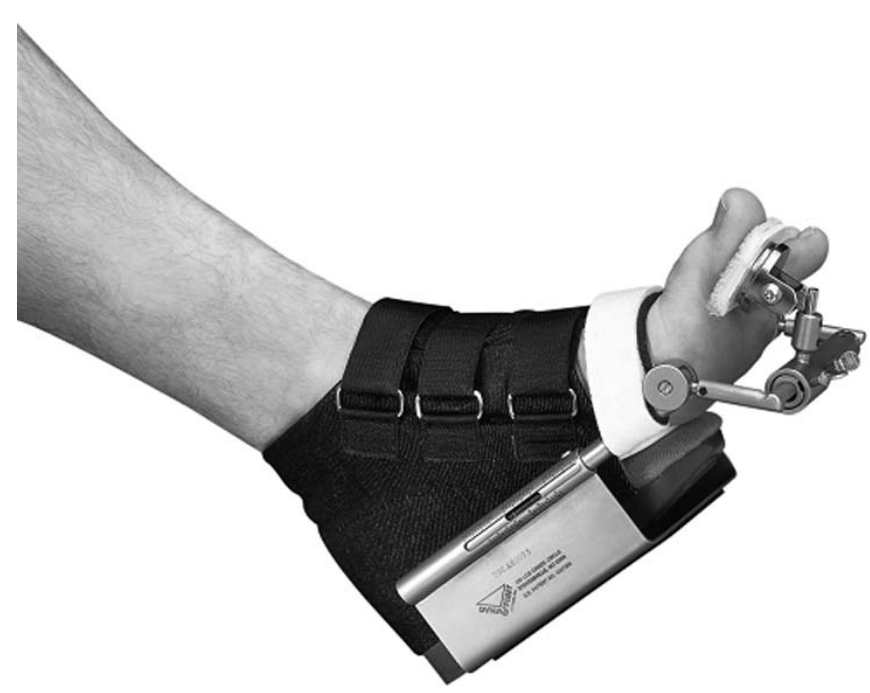

Figure 1 Metatarsophalangeal Dynasplint Extension System.

The procedure of utilizing low-load, prolongedduration stretch has been effective in reducing contracture in similar conditions of reduced $\mathrm{ROM}^{3,12,13}$ because it does have biomechanically appropriate alignment with measurable tension. Dynamic splinting used the protocol of low-load, prolonged-duration stretch to achieve significant time at their end range of motion. As the range of motion progresses, the calibrated tension of the dynamic splint is increased to continue stretching at the end range. The purpose of this retrospective cohort study was to examine the efficacy of dynamic splinting for treating HL.

\section{Methods}

\section{Patients}

Case histories were retrospectively acquired for 61 patients who had been diagnosed with HL which continued following contusion or surgery. These patients were treated for one or more months with dynamic splinting.

\section{Intervention}

Patients' initial introduction to the Metatarsophalangeal Dynasplint System (MDS) [Dynasplint Systems, Inc., Maryland, USA], included customized fitting (patient's foot size and varying degrees of hallux edema) and training on donning and doffing of the devices. (Fig. 1) Verbal and written instructions were provided throughout the duration of treatment for safety, general wear and care, and tension setting goals based on patient tolerance.

Each patient was instructed to start wearing the MDS initially for 30 minutes, three times a day while seated at a tension setting of \#1 (0.10 foot pound of torque). This frequency, intensity, and duration were for acclimatization to the system. The patients were then instructed to increase each wear by ten minutes each day (i.e. $30 \mathrm{~min}$ tid (three times a day, latin for ter in die), $40 \mathrm{~min}$ tid, $50 \mathrm{~min}$, tid, $60 \mathrm{~min}$ tid), until he/she was comfortable wearing the unit for a total of 3 hours each day.

After each patient was comfortable wearing the unit for a total of 3 hours per day, for three days, the patient was instructed to increase the tension one increment every week until reaching setting \#4 (0.7 foot pounds of torque). If excess fatigue followed a session (soreness for more than 15 minutes) the patient was instructed to decrease the tension one half a setting for two days until comfortable wearing for 60 minutes per session and then recalibrate at the higher setting. Tracking of patient compliance was accomplished with a dairy, which was submitted to the attending clinicians monthly.

\section{Statistics}

The dependent variable in this study was the change in dorsiflexion at the metatarsal joint of the great toe, and the independent variables were the patient categories of bunionectomy vs. cheilectomy vs. contusion. Statistical data analysis was accomplished using a one-way analysis of variance (ANOVA) on data collected after one month's use of the MDS. 


\section{Dynamic Splinting and Metatarsophalangeal Joint Extension}

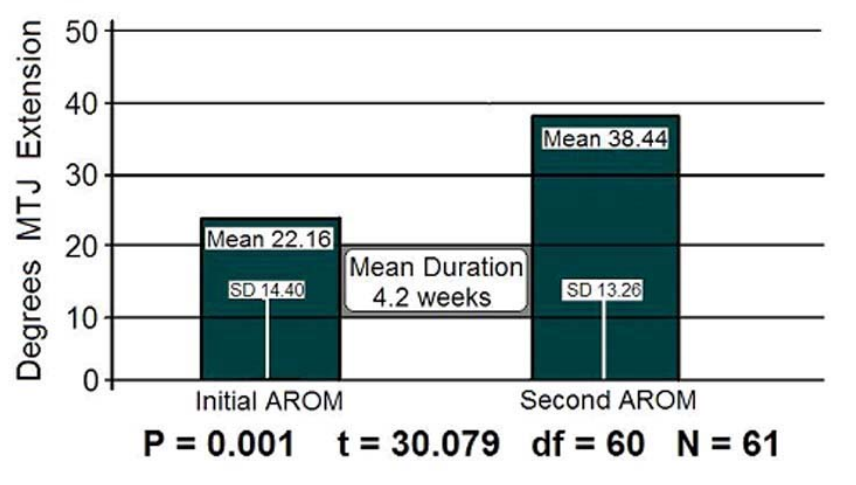

Figure 2 Changes in MTJ Extension.

Results

After a mean duration of 4.2 weeks, the ANOVA showed a significant change for all groups $(\mathrm{N}=61$, $\mathrm{P}<0.001, \mathrm{~T}=30.079, \mathrm{DF}=60)$. However, there was not a significant difference between groups $(\mathrm{P}>0.05)$. (Fig. 2)

\section{Discussion}

The cause for postoperative contracture is hypothesized to be due to excess arthrofibrosis and position due to pain. $^{10}$ Secondary treatment procedures include surgical realignment as done with the Kalish Osteotomy. If mobility is not regained and pain is not reduced, then arthrodesis is considered as a final salvage procedure. ${ }^{11}$ However, a non-invasive procedure for contracture reduction is usually more desirable. A pilot study recently showed the efficacy of dynamic splinting for regaining flexion in the great toe $^{3}$, but this is the first study to examine changes in hallux extension, dorsiflexion at the metatarsal joint, following dynamic splinting.
The purpose of this retrospective cohort study was to examine the efficacy of dynamic splinting for treating hallux limitus. Dynamic splinting used as a home therapy, provided an additional 90 hours per month of stretching at end range for each patient. This was accomplished with controlled, calibrated, changeable tension which adapted to gains in ROM, keeping the joint at end range.

\section{Conclusion}

This retrospective cohort study showed a statistically significant difference in ROM following use of the MDS $(\mathrm{P}<0.0001)$, with patients gaining a mean $73 \%$ increase in dorsiflexion at the metatarsal joint of the great toe in just one month. MDS utilizes a biomechanical adaptation to achieve a physiological change in contracture reduction of the connective tissue. Patients in this study received a mean 90 hours of end range stretching in home therapy resulting in regained ROM, eliminating the need for secondary surgical procedures to reduce the contracture. ${ }^{11}$ 


\section{References}

1. Goucher NR, Coughlin MJ. Hallux metatarsophalangeal joint arthrodesis using dome-shaped reamers and dorsal plate fixation: a prospective study. Foot Ankle Int 27(11):869 - 76, 2006.

2. Hockenbury RT. Forefoot problems in athletes. Med Sci Sports Exerc 31: S448 - 58, 1999.

3. Willis B, John M. Dynamic Splinting Increases Flexion for Hallux Rigidus (Pilot Study). Biomechanics 14(9), pg49 - 53, 2007. 4. Brodsky JW, Baum BS, Pollo FE, Mehta H. Prospective gait analysis in patients with first metatarsophalangeal joint arthrodesis for hallux rigidus. Foot Ankle Int. 28(2):162 - 5, 2007. 5. Talarico LM, Vito GR, Goldstein L, Perler AD. Management of hallux limitus with distraction of the first metatarsophalangeal joint. J Am Podiatr Med Assoc 95(2):121 - 9, 2005.

6. Payne C, Chuter V, Miller K. Sensitivity and Specificity of the Functional Hallux Limitus Test to Predict Foot Function. $J$ Am Podiatr Med Assoc 92: 269 - 271, 2002.

7. Kennedy JG, Chow FY, Dines J, Gardner M, Bohne WH. Outcomes after interposition arthroplasty for treatment of hallux rigidus. Clin Orthop Relat Res 445:210 - 5, 2006.

8. DeFrino PF, Brodsky JW, Pollo FE, Crenshaw SJ, et al: First metatarsophalangeal arthrodesis: a clinical, pedobarographic and gait analysis study. Foot Ankle Int 23(6): 496 - 502, 2002.

9. Canseco K, Long J, Marks R, Khazzam M, Harris G.

Quantitative characterization of gait kinematics in patients with hallux rigidus using the Milwaukee foot model. J Orthop Res 30, 1 $-9,2007$

10. Becher C, Kilger R, Thermann H. Results of cheilectomy and additional microfracture techniques for the treatment of hallux rigidus. Foot Ankle Surg (10): 155-160, 2005.

11. Lau JT, Daniels TR: Outcomes following cheilectomy and interpositional arthroplasty in hallux rigidus. Foot Ankle Int 2001 22 (6): $462-70,2001$.

12. Hepburn, G. Contracture and Stiff Joint Management with Dynasplint. J Ortho Sports Phys Ther 8 (10): 498 - 504, 1987.

13. Willis B. Dancers restore knee flexion through dynamic splinting. Biomechanics 15 (1), 49 - 54, 2008.

\section{Acknowledgements}

The authors thank Dr. Ram Shanmugam, biostatistics professor at Texas State University, San Marcos, TX for performing statistical analysis.

\section{Conflict of Interest}

Regarding conflict of interest, no extramural funding was used in this study. Dr. Kalish is an educator for the parent company of Dynasplint Systems and he did not receive any funding for this study. Dr. Willis is employed by Dynasplint Systems, Inc. and has no ownership or stock in this company.

\section{Equipment}

Dynasplint ${ }^{\circledR}$ Systems, Inc. 770 Ritchie Highway, Suite W21

Severna Park, MD 21146

SPSS Inc. (Base Statistical System)

233 S. Wacker Drive, 11 th floor

Chicago, IL 60606-6307 